\section{A telecolaboração como facilitadora do diálogo intercultural no Instituto Federal Catarinense}

Telecollaboration as a facilitator of the intercultural dialogue at Instituto Federal

Catarinense

Rodrigo SCHAEFER (IFC-Brusque/Blumenau) rodrigo.schaefer@ifc.edu.br

Christiane HEEMANN (UNIVALI) chrisheemann@gmail.com

Recebido em: 25 de jan. de 2021. Aceito em: 25 de ago. de 2021.

\title{
00000000000
}

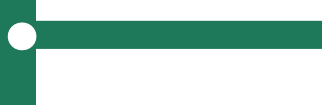

SCHAEFER, Rodrigo; HEEMANN,

Christiane. A telecolaboração como

facilitadora do diálogo intercultural no Instituto Federal Catarinense.

Entrepalavras, Fortaleza, v. 11, n. 3, e2228, p. 170-187, set.-dez./2021. DOI: 10.22168/2237-6321-32228.

Resumo: O impacto das Tecnologias de Informação e Comunicação (TICs), tendo em vista o grande número de educadores e alunos que têm aderido à sua utilização, é tido como revolucionário. Atividades telecolaborativas, possibilitadas pela utilização das TICs, permitem, mediante o contato entre pessoas de diferentes países e culturas, a prática do inglês. Este artigo tem como objetivo discutir o potencial da telecolaboração como facilitadora do diálogo intercultural nas aulas de inglês do Instituto Federal Catarinense (IFC). Este estudo conta com o aporte teórico de pesquisadores como O'Dowd (2006, 2019), Telles (2015), Byram (2016), Sequeira (2018), entre outros. Os resultados apontam que a telecolaboração, dada a sua natureza dialógica (O'DOWD, 2003, 2021; THORNE, 2006; BELZ, 2007; HELM, 2016), propicia a discussão em inglês de vários assuntos entre estudantes de diferentes referenciais culturais. Diante disso, sugerimos que as atividades telecolaborativas estão a serviço dos professores, que podem mediar o encontro entre estudantes, línguas e culturas, integrando práticas e contatos mais significativos na sua ação pedagógica.

Palavras-chave: Telecolaboração. Diálogo intercultural. Ensino e aprendizagem de inglês. Educação Profissional e Tecnológica. Instituto Federal Catarinense. 
Abstract: The impact of Information and Communication Technologies (ICTs), bearing in mind the large number of educators and students who have been using them, is seen as revolutionary. Telecollaborative activities, made possible by the use of ICTs, allow, through the contact between people from different countries and cultures, the practice of English. This article seeks to discuss the potential of telecollaboration in facilitating the intercultural dialogue in the English classes at Instituto Federal Catarinense (IFC). This study includes scholars' theoretical contributions such as O'Dowd (2006, 2019), Telles (2015), Byram (2016), Sequeira (2018), among others. The results show that, due to its dialogical nature (O'DOWD, 2003, 2021; THORNE, 2006; BELZ, 2007; HELM, 2016), telecollaboration enables the discussion in English of various subjects among students of different cultural references. Therefore, we suggest that telecollaborative activities are at the service of teachers, who can mediate the encounter between students, languages and cultures, integrating more meaningful practices and contacts into their pedagogical action.

Keywords: Telecollaboration. Intercultural Dialogue. English Teaching and Learning. Professional and Technological Education. Catarinense Federal Institute.

\title{
Considerações iniciais
}

As Tecnologias de Informação e Comunicação (doravante TICs), tais como wikis, plataformas online e aplicativos para videoconferência, têm exercido influência sobre o processo de ensino e aprendizagem em geral. Conforme Alcici (2014), o uso das TICs "contribui para expandir o acesso à informação atualizada, permite estabelecer novas relações com o saber que ultrapassam os limites dos materiais instrucionais previamente preparados e sob o controle dos educadores, favorece a interatividade, permitindo a comunicação com o mundo" (p. 14). Evidentemente, de acordo com o Digital Education Action Plan ${ }^{1}$ (21-27) (COMISSÃO EUROPEIA, 2020) (DEAP), o impacto potencial das TICs é tido como revolucionário, inclusive se considerarmos o grande número de educadores e alunos que vêm aderindo à sua utilização. Almeida (2014) explica que a implementação das TICs no contexto escolar permite os seguintes aspectos:

\begin{abstract}
Acesso a contextos variados; possibilidade de pesquisas na área de interesse; discussão de conteúdos mediante a disponibilidade de informação na rede; motivação; aproximação entre o conteúdo e a realidade do aluno; participação colaborativa; relação entre prática e teoria; compartilhamento de experiências; desenvolvimento de potencialidades; percepção de outras linguagens que não a linguagem convencional ensinada na escola. (ALMEIDA, 2014, p. 39-40)
\end{abstract}

Para Sehnem e Luna (2013), no contexto brasileiro do ensino de línguas estrangeiras, muitas vezes os estudantes não desenvolvem as habilidades necessárias para se comunicar suficientemente bem

\footnotetext{
1 "Plano de Ação de Educação Digital".
} 
v. $11(3)$ 170-187 set-dez 2021 em outras línguas. Os autores complementam que a competência comunicativa almejada é vista como um diferencial no mercado de trabalho, bem como um objetivo traçado por muitos professores de línguas. Na visão de Canale (1995) e Ramiro (2007), a competência comunicativa está relacionada com a união do conhecimento linguístico e da competência discursiva, fazendo com que os estudantes se expressem de forma inteligível tanto oralmente quanto por escrito.

Atividades telecolaborativas, facilitadas pela utilização das TICs, permitem, além da prática das quatro habilidades linguísticas leitura, escrita, fala e audição -, o contato entre pessoas de diferentes países e culturas (O'DOWD, 2013, 2016). Segundo O'Dowd (2013), a telecolaboração se refere à

aplicação de ferramentas de comunicação online para encontro de aprendizes de línguas em locais geograficamente distantes para desenvolver suas habilidades em língua estrangeira e sua competência intercultural através de tarefas colaborativas e trabalho de projeto. (O'DOWD, 2013, p. 123, tradução nossa²).

O termo telecolaboração foi criado por Mark Warschauer

(1996) na sua publicação Telecollaboration and the Foreign Language Learner, e uma edição especial da revista Language Learning \& Technology foi dedicada em 2003 à essa área então emergente. A telecolaboração era vista pelos estudantes, por Warschauer (1996) e outros estudiosos como facilitadora da aprendizagem de línguas, dado que diferentes procedimentos metodológicos vinham sendo utilizados e se mostraram adaptáveis a vários objetivos pedagógicos e contextos de aprendizagem.

Diante do potencial da telecolaboração para a prática de habilidades linguísticas, o presente estudo tem como objetivo discutir o potencial da telecolaboração como facilitadora do diálogo intercultural nas aulas de inglês do Instituto Federal Catarinense (IFC).

Este texto está dividido em cinco partes. A primeira se concentrou no objetivo, assim como em uma breve contextualização deste estudo. A segunda parte, por sua vez, trará aportes teóricos sobre o diálogo intercultural permitido pela telecolaboração. Em seguida, o foco recairá sobre os procedimentos metodológicos adotados, e, na quarta parte, será discutido o modo pelo qual as atividades telecolaborativas desenvolvidas com aprendizes de inglês do IFC abriram espaço para

\footnotetext{
${ }^{2}$ Citação original: "the application of online communication tools to bring together classes of language learners in geographically distant locations to develop their foreign language skills and intercultural competence through collaborative tasks and project work".
} 
o diálogo intercultural. Por último, teceremos, na quinta parte, considerações finais.

\section{O diálogo intercultural pela telecolaboração}

Segundo Thorne (2006), a telecolaboração dispõe de diferentes oportunidades para o diálogo intercultural ${ }^{3}$, como pequenos grupos de trabalho, troca de informações entre todos os alunos da sala e trabalhos em duplas. Para o autor, atividades "em torno da informação e mídia (literatura, filmes, textos acadêmicos) e atividades colaborativas, interpretativas e investigativas" ${ }^{4}$ (p. 7) podem ser integradas em projetos telecolaborativos.

Dada a natureza interativa das atividades colaborativas, Belz (2007) defende que a telecolaboração pode ser caracterizada como etnográfica, dialógica e crítica. Etnográfica porque os estudantes podem observar, analisar e interpretar o comportamento de seus parceiros online. Dialógica no sentido de que os discursos dos estudantes surgem a partir da interação com os outros. Finalmente, crítica uma vez que "os aprendizes não são receptáculos passivos do conhecimento recebido [...], mas sim participantes ativos em um processo dinâmico de construção do conhecimento"s (p. 138).

A oportunidade para o diálogo, como mencionada por Thorne (2006) e Belz (2007), é também sinalizada por outros pesquisadores que lidam com telecolaboração. Por exemplo, Helm (2016, p. 153) ressalta que, através do diálogo colaborativo, "os participantes exploram identidades e diferenças, experiências pessoais e emoções, as quais contribuem para a consciência de si e dos outros" ${ }^{\prime}$, ao passo que O'Dowd (2003, p. 133) explica que atividades envolvendo o diálogo permitem que "os alunos expressem suas ideias e depois as esclareçam e redefinam através do feedback e de outras perspectivas às quais estão expostos" "7. Do mesmo modo que O'Dowd (2003), Tella e Mononen-Aaltonen (1998) entendem

\footnotetext{
3 Na visão de Thorne (2006) e Schaefer (2020, 2021), o diálogo e outras formas de interação podem fomentar o desenvolvimento da competência comunicativa intercultural entre membros de grupos culturais e linguísticos diferentes.

${ }^{4}$ Citação original: "around shared information and media (literature, films, scholarly texts) and collaborative, interpretative and investigative activities".

${ }^{5}$ Citação original: "learners are not passive receptacles of received knowledge [...], but rather active participants in a dynamic process of knowledge construction".

${ }^{6}$ Citação original: "participants explore identities and difference, personal experience and emotions, which contribute to awareness of self and others".

${ }^{7}$ Citação original: enables "learners to express their ideas and then to clarify and redefine them through feedback and through the other perspectives to which they are exposed".
} 
V. 11 (3) 170-187 set-dez 2021

que momentos de diálogo em contextos telecolaborativos possibilitam "a interação entre si e o outro e a incorporação do horizonte conceitual do último à sua própria perspectiva"s (p. 14). Finalmente, Veloso e Almeida (2009) argumentam que o diálogo leva os estudantes a terem um controle maior sobre a sua aprendizagem.

A seguir, a partir do trabalho de O'Dowd e Lewis (2016), apresentamos três exemplos de projetos de telecolaboração que visam promover o contato intercultural. O primeiro deles é o projeto The Cultnet Intercultural Citizenship, cujo objetivo é compreender como a educação para a cidadania pode ser incluída no ensino e aprendizagem de língua estrangeira através de subprojetos (BYRAM, 2016). O segundo, The Cultura Exchange Programme, pretende possibilitar o contato intercultural entre alunos de diferentes países (FURSTENBERG, 2016). O terceiro projeto é o Teletandem Brasil: Línguas estrangeiras para todos (doravante TTB) (TELLES; VASSALLO, 2006; TELLES, 2015). Desenvolvido em uma universidade pública estadual brasileira e criado em 2006, tem como objetivo permitir o contato entre estudantes brasileiros e de outros países.

Para Byram (1997), a interculturalidade9 está relacionada com a habilidade de utilizar a língua nos diálogos interculturais. O autor propõe cinco componentes para o desenvolvimento de tal habilidade: (1) atitudes, que se referem a uma "disposição para suspender crenças com relação a seus próprios significados e comportamentos, e analisá-los do ponto de vista dos outros com quem o indivíduo está interagindo"10 (p. 34); (2) saberes, no sentido de obter conhecimentos de outras culturas e de si mesmo nas interações sociais; (3) habilidades de descobertas e interação, que têm a ver com "a habilidade de adquirir novos conhecimentos de uma cultura e práticas culturais e a habilidade para usar conhecimentos, atitudes e habilidades sob as limitações da comunicação e interação em tempo real" "11 (p. 61); (4) habilidades de interpretação e relacionamento, que significa saber interpretar um documento ou um evento de outra cultura e relacioná-lo com sua

\footnotetext{
${ }^{8}$ Citação original: "interaction between self and other and the incorporation of the latter's conceptual horizon to one's own perspective".

9 Na realidade, Byram (1997) utilizou o termo "competência comunicativa intercultural", e não "interculturalidade".

${ }^{10}$ Citação original: "willingness to suspend belief in one's own meanings and behaviors, and to analyze them from the viewpoint of the others with whom one is engaging".

${ }^{11}$ Citação original: "the ability to acquire new knowledge of a culture and cultural practices and the ability to operate knowledge, attitudes and skills under the constraints of real-time communication and interaction".
} 
própria cultura; por último, (5) consciência cultural crítica, que está relacionada com o exame e a interpretação de maneira crítica acerca de diferenças culturais. De acordo com Byram, Gribkova e Starkey (2002), a referida consciência pode ajudar os aprendizes de línguas a "agir em uma base de novas perspectivas"12 (p. 29).

Praticamente todas as conceitualizações de interculturalidade transcendem a ideia de meramente obter conhecimento sobre outras culturas, já que esse tipo de conhecimento não é suficiente para um indivíduo ser interculturalmente competente. Nesse sentido, O’Dowd (2006) destaca que

Além de conhecimento sobre a outra cultura e atitudes de abertura e interesses por outras culturas, a interação intercultural eficaz inclui as habilidades de ser capaz de descobrir e entender o significado simbólico que é atribuído aos comportamentos em diferentes culturas. (O'DOWD, 2006, p.86, tradução nossa ${ }^{13}$ )

Kramsch (2006) argumenta que, nos encontros interculturais, para além de uma competência turística, caracterizada como troca de informações com falantes nativos, é necessário o desenvolvimento da interculturalidade. Deardorff (2004) e Byram (2017), por seu turno, afirmam que tal desenvolvimento implica habilidades e atitudes para interagir de forma bem-sucedida com pessoas de diferentes culturas. Com base em tais definições, defendemos que a interculturalidade pode preparar os indivíduos para o desempenho de diferentes ações na comunidade assim como de atividades futuras enquanto cidadãos.

No que segue, apresentamos dois estudos que incluíram o diálogo intercultural em suas atividades telecolaborativas. O primeiro deles, intitulado Understanding the 'other side': intercultural learning in a Spanish-English e-mail exchange ${ }^{14}$, de O'Dowd (2003), teve como objetivo analisar a troca de $e$-mails entre cinco pares de aprendizes universitários de inglês e espanhol. O'Dowd, então professor de espanhol dos cinco participantes de seu estudo, aplicou sua pesquisa por meio de um projeto entre uma universidade da Espanha e uma da Inglaterra. Para alcançar

\footnotetext{
${ }^{12}$ Citação original: "to act on a [sic] the basis of new perspectives".

${ }^{13}$ Citação original: "Apart from knowledge of the target culture and attitudes of openness toward and interest in other cultures, effective intercultural interaction includes the skills of being able to discover and understand the symbolic meaning that is attributed to behaviors in different cultures".

${ }^{14}$ Em português: Compreendendo o 'outro lado': aprendizagem intercultural por $e$-mail em espanhol e inglês.
} 
v. $11(3)$ 170-187 set-dez 2021

o objetivo supracitado, o autor adotou uma abordagem etnográfica, a qual envolve técnicas como observação, questionários e entrevistas. Os resultados mostraram que aspectos como "questões sobre o estrangeiro" e "enxergando o mundo através dos olhos do outro" constituíram terreno fértil para a discussão de assuntos interculturais entre os participantes.

O segundo estudo, Negotiation of cultural issues equated to nation in e-mail exchanges ${ }^{15}$ (SCHAEFER, LUNA, 2016), delineou o objetivo de compreender como assuntos associados com uma perspectiva nacional foram negociados e construídos dialogicamente em trocas de $e$-mail. Giulia e Carlos $^{16}$ interagiam uma vez por semana, pelo período de cinco meses. Este, brasileiro e professor de inglês, pretendia desenvolver suas habilidades na língua italiana. Giulia, por sua vez, era italiana e seu objetivo era aprender a se comunicar em língua inglesa. Para atingir o objetivo referido, foram analisados dois episódios culturais: o primeiro deles sobre dança latina e o segundo atrelado à suposição, por parte de Giulia, de que o povo italiano não obedece às leis nacionais. Com base nos resultados da análise dos dados, foi possível perceber que (1) os tópicos discutidos na interação por $e$-mail estiveram vinculados a uma visão de cultura enquanto construto nacional e; (2) a negociação dos significados foi uma constante, tanto no que se refere aos temas culturais abordados quanto a questões linguísticas.

Assim como as pesquisas conduzidas por O'Dowd (2003) e Schaefer e Luna (2016), o presente estudo está centrado em interação por e-mail. Portanto, levando em consideração que esses estudos evidenciaram a possibilidade do diálogo intercultural e de aprendizagem de línguas na telecolaboração, nosso objetivo, conforme já mencionado, é discutir o potencial da telecolaboração como facilitadora do diálogo intercultural nas aulas de inglês do IFC. Diante disso, a seção seguinte descreverá as atividades realizadas.

\section{Interações telecolaborativas entre alunos do IFC e alunas de uma universidade pública brasileira: procedimentos metodológicos}

O presente estudo está vinculado ao Projeto de Conversação em Inglês do IFC campus Brusque, coordenado por um dos autores (doravante professor do IFC), que tem como objetivo promover a prática do inglês e o diálogo entre servidores, estudantes, docentes e

\footnotetext{
${ }^{15}$ Em português: Negociação de questões culturais equiparadas a nação em trocas de $\boldsymbol{e}$-mail.

${ }^{16}$ Nomes fictícios.
} 
administrativos do IFC e, do mesmo modo, da comunidade externa em geral. Para tal, o projeto utiliza-se de recursos como o Google Meet, vídeos, letras de músicas, trechos de filmes, entre outros.

Ao longo de cerca de um mês, no segundo semestre de 2019, o professor do IFC integrou o referido projeto na disciplina ministrada por ele - Inglês Instrumental. Esta, que contava com 20 alunos matriculados, compõe a grade curricular do curso Tecnologia de Redes de Computadores (Ensino Superior). Os alunos interagiram com cinco alunas do Curso de Letras $^{17}$ da Universidade do Estado da Bahia (doravante UNEB), campus IV (Jacobina), matriculadas no componente de Inglês - nível Intermediário III.

Conforme será explicado no próximo parágrafo, as atividades telecolaborativas do presente estudo ocorreram mediante a escrita de um texto revisado por pares. Segundo Bolzan (2016), a prática da revisão por pares possibilita aos aprendizes de línguas a interação colaborativa, o que permite também a eles a co-construção do conhecimento no processo de aprendizagem de língua. A autora acrescenta que, ao participar da prática mencionada, "o aprendiz estará desenvolvendo sua autonomia" (BOLZAN, p. 43) enquanto aprendiz e escritor.

As atividades telecolaborativas do nosso estudo foram organizadas pelo professor do IFC e pela professora ${ }^{18}$ da UNEB, e duraram cerca de um mês. Mais especificamente, tais atividades consistiram na escrita de um texto redigido em inglês (entre 15 e 20 linhas) por parte dos alunos do IFC, que trabalharam em duplas, e encaminhado por e-mail ${ }^{19}$ para as cinco alunas do Curso de Letras da UNEB, e com cópia para o professor do IFC e para a professora da UNEB. Os textos solicitados precisavam apresentar informações sobre os alunos (por exemplo, nome e idade), o campus onde estudavam, o curso que faziam, a cidade onde moravam e a ocupação que exerciam, assim como atividades rotineiras e de lazer.

Ao receberem o primeiro texto dos alunos do IFC, as alunas do Curso de Letras da UNEB realizaram os seguintes procedimentos: a) responderam, em formato de texto encaminhado por e-mail para os alunos do IFC, às informações gerais que eles abordaram e, além

\footnotetext{
${ }^{17}$ Professoras em formação.

${ }^{18}$ Agradecemos à professora Dra. Juliane Regina Trevisol por ter aceito o convite de desenvolver as atividades telecolaborativas descritas no presente estudo.

${ }^{19}$ Apesar de outras tecnologias, houve a escolha pelo $\boldsymbol{e}$-mail por ser uma forma segura e rápida de interação, bem como organizada de manter os textos enviados e recebidos arquivados.
} 
v. 11 (3) 170-187 set-dez 2021 disso, elas incluíram no referido texto cinco perguntas relacionadas a tais informações (a fim de conhecer mais sobre onde eles viviam, por exemplo); e b) teceram comentários acerca da qualidade da redação, sugeriram ajustes e corrigiram desvios linguísticos. É conveniente precisar que embora as alunas da UNEB tenham sugerido ajustes gerais com relação à produção textual dos alunos do IFC, alertamos os estudantes de ambas as instituições sobre a importância de a atenção das interações por e-mail centrarem-se na construção de significados (inter)culturais. Em sintonia com O'Dowd (2003) e Helm (2016), é possível, mediante o diálogo intercultural, abordar aspectos culturais bem como expor ideias e experiências pessoais. Posto isto, haja vista que o enfoque principal das interações não recaiu sobre aspectos linguístico-gramaticais, percebemos que os alunos do IFC se sentiram à vontade para compartilhar informações e atividades rotineiras, o que facilitou o diálogo intercultural segundo O'Dowd (2003) e Helm (2016).

Devido à diferença do número de alunos nos dois grupos, as cinco alunas do Curso de Letras da UNEB desenvolveram as atividades individualmente. Em geral, cada aluna interagiu com duas duplas de alunos do IFC. Após receberem os textos, as alunas redigiram suas respostas individualmente, com comentários e correções dos textos, e, em um segundo momento, e antes mesmo de enviarem novamente o texto por $e$-mail aos alunos do IFC, elas se reuniram em grupo com a professora da UNEB a fim de discutir e revisar colaborativamente alguns dos elementos registrados.

Foi solicitada também aos alunos do IFC uma segunda versão do texto, a fim de que pudessem melhorar a qualidade da redação com base nos elementos anotados/corrigidos pelas alunas da UNEB. Dessa forma, o professor do IFC e a professora da UNEB objetivaram também a habilidade de reescritura dos alunos do IFC. A fim de ilustrar a organização da proposta, o Quadro 1 apresenta o cronograma das atividades desenvolvidas. 
Quadro 1 - Cronograma de Atividades

\begin{tabular}{|l|l|}
\hline $\begin{array}{l}\text { Data limite } \\
\text { de entrega }\end{array}$ & \multicolumn{1}{|c|}{ Descrição da atividade } \\
\hline $17 / 11 / 19$ & $\begin{array}{l}\text { Cada dupla de alunos do IFC enviou o texto discorrendo sobre sua } \\
\text { rotina, sua cidade e seus hobbies para uma das alunas do Curso de } \\
\text { Letras da UNEB. }\end{array}$ \\
\hline $19 / 11 / 19$ & $\begin{array}{l}\text { As alunas da UNEB, a fim de realizar o processo de revisão do texto } \\
\text { dos alunos do IFC, reuniram-se com sua professora para discutir suas } \\
\text { anotações. }\end{array}$ \\
\hline $24 / 11 / 19$ & $\begin{array}{l}\text { Cada aluna da UNEB retornou o texto para suas respectivas duplas do } \\
\text { IFC com comentários e sugestões de ajustes. Além disso, cada aluna } \\
\text { elaborou cinco perguntas referentes a atividades rotineiras e outras } \\
\text { com base no conteúdo do texto escrito pelos alunos do IFC. }\end{array}$ \\
\hline $01 / 12 / 19$ & $\begin{array}{l}\text { As duplas do IFC enviaram novamente, e pela última vez, o texto para as alunas } \\
\text { da UNEB com as solicitações de ajustes e de reconstrução do texto atendidas, } \\
\text { incluindo também as respostas às questões elaboradas anteriormente. }\end{array}$ \\
\hline $08 / 12 / 19$ & $\begin{array}{l}\text { Cada aluna da UNEB retornou, mais uma vez, o texto para suas respectivas } \\
\text { duplas do IFC em resposta às correções e ajustes feitos nos textos, } \\
\text { apresentando, ademais, comentários gerais e sugestões de escrita. }\end{array}$ \\
\hline
\end{tabular}

Fonte: Próprios autores, 2021.

$\mathrm{Na}$ última semana da condução das atividades telecolaborativas, o professor do IFC e a professora da UNEB convidaram seus respectivos grupos de alunos a partilharem oralmente, em sala de aula, sua experiência referente à participação em tais atividades. A professora da UNEB, a título de ilustração, pediu que suas cinco alunas apresentassem sua opinião sobre as atividades e o que fora positivo e desafiador ao longo do desenvolvimento do projeto. O professor do IFC, por sua vez, solicitou a seus alunos que comentassem sobre aspectos linguístico-culturais. Essas percepções foram gravadas em áudio.

Dito isto, no presente estudo analisamos as falas de duas alunas: Antônia (do IFC) e Madalena ${ }^{20}$ (da UNEB). Esta tinha 22 anos e estava em estágios mais avançados do aprendizado de inglês, ao passo que aquela tinha 21 anos de idade e estava em estágios iniciais.

Na seção seguinte, abordamos, com base nas percepções de Antônia e Madalena, a experiência telecolaborativa.

\section{Oportunidade para o diálogo intercultural segundo as alunas}

Os comentários das alunas enfatizaram, de um modo geral, que a atividade telecolaborativa proporcionou uma experiência bastante relevante. A possibilidade de troca cultural, bem como de aprendizado

${ }^{20}$ Nomes fictícios para salvaguardar a identidade das alunas. 
v. 11 (3) 170-187 set-dez 2021

da língua inglesa, referente a aspectos gramaticais e lexicais, foram os elementos mais recorrentes. Com efeito, Sehnem e Schaefer (2018) sublinham que interações telecolaborativas propiciam uma prática significativa da língua em estudo, ao passo que O'Dowd (2007, 2013, 2019, 2021) entende que a telecolaboração, na área do ensino e aprendizagem de línguas estrangeiras, permite a aprendizagem e o diálogo entre pessoas de diferentes culturas.

Mais especificamente, as alunas ressaltaram que a atividade ofereceu uma ocasião oportuna para entrarem em contato com estudantes de outro estado do Brasil, com quem puderam discutir diferentes assuntos e desenvolver as habilidades de escrita em inglês. Por exemplo, uma das alunas afirmou o seguinte: "bem legal esse contato que a gente teve, abrindo a porta para conhecer alguém de outro estado e ver que a gente tem coisa em comum". A oportunidade para o contato enfatizada pela aluna vai ao encontro do estudo de Schaefer e Heemann (2019). No estudo em questão, identificou-se que atividades telecolaborativas facilitam a prática da língua estudada pelos alunos, bem como o diálogo entre pessoas de diferentes culturas. Semelhantemente, o estudo de O'Dowd (2003) e o de Schaefer e Luna (2016), revistos anteriormente, mostraram que as interações telecolaborativas permitiram que os aprendizes de línguas discutissem diferentes assuntos. Ainda, autores como Byram (2016), Furstenberg (2016), Helm (2016), Sequeira (2018) e Schaefer (2019a, 2019b, 2020, 2021) explicam que a telecolaboração constitui terreno fértil para o contato entre pessoas de diferentes culturas.

A seguir, o Excerto 1 elucida como elementos culturais advindos da atividade telecolaborativa foram relevantes para Antônia.

Antônia: Nossa interação é com a Madalena. Ela comentou do clima que é muito quente, variado, quente e frio, e ela falou da natureza, que tem cachoeira, coisa bem legal. Nós também comentamos da nossa localidade perto da praia, perto da cachoeira, perto da serra, perto da neve, próximo, então assim comentamos do nosso local e ela ficou bem interessada também em conhecer um dia nossa região, e foi bem legal.

Professor do IFC: Ah, olha, então elas ficaram interessadas e vocês também ficaram interessados em conhecer a região lá? (Excerto 1, dezembro de 2019).

Como é possível perceber, a aluna pôs em relevo a oportunidade de discutir referentes culturais, nesse caso relacionados com o clima dos dois estados em tela (Santa Catarina e Bahia). Efetivamente, a 
oportunidade citada converge com O'Dowd (2006, 2019, 2021), Byram (1997) e Rodrigues (2013), no sentido de que conhecimento e interesse sobre outras culturas é essencial nos contatos interculturais. De modo similar, Byram (1997, 2016), Byram, Gribkova e Starke (2002), O’Dowd (2003, 2006, 2013, 2021) e Barrett et al. (2013) afirmam que é necessário "abrir-se para o outro" e comparar diferentes aspectos culturais nos encontros entre pessoas de diferentes culturas, o que, conforme o Excerto 1, sucedeu entre as alunas.

No que tange à possibilidade de discutir diferentes assuntos através de atividades telecolaborativas, em sintonia com o que O'Dowd (2003) e Schaefer e Luna (2016) nos lembraram anteriormente, o excerto a seguir descreve a percepção positiva em relação a tais atividades por parte de Madalena.

Madalena: Eu achei bem legal. Primeiro porque a gente nunca fez algo assim, então foi uma experiência legal e também a gente acabou conhecendo mais da cultura deles. Acho que todo mundo acabou aprendendo alguma coisa nova com eles.

Professora da UNEB: O quê, por exemplo?

Madalena: Ah, aqueles negócio lá, do, do Papai Noel do mal, que não sei falar o nome, que eu achei bem sinistro. (Excerto 2, dezembro de 2019)

Nesse excerto, Madalena esclareceu que as atividades telecolaborativas possibilitaram o conhecimento de aspectos culturais relacionados ao estado dos alunos do IFC, isto é, sobre a tradição do Papai Noel do Mato, ou Pelznickel21. Neste sentido, Byram (1997) defende a ideia de que nos contatos interculturais é indispensável discutir assuntos diversos, tais como instituições, produtos culturais e tradições, sob a perspectiva de pessoas que vivem no contexto cultural, o que permite afirmar que o diálogo entre a aluna do IFC e a da UNEB proporcionou não apenas a prática da língua inglesa, mas também das habilidades de descoberta e interação (BYRAM, 1997). Sob esta ótica, é possível dizer que as atividades telecolaborativas, em contraste com alguns contextos de ensino e aprendizagem de línguas que "encerram o diálogo e esterilizam o debate" 22 (KELLY, 2004, p. xii), se mostraram relevantes para as alunas.

\footnotetext{
${ }^{21}$ Para maiores informações relacionadas a este expressão cultural, acesse o sítio https:// turismo.guabiruba.sc.gov.br/equipamento/index/codEquipamento/6203 .

${ }^{22}$ Citação original: "close down dialogue and sterilise debate".
} 
V. $11(3)$

170-187

set-dez

2021

Após análise referente ao diálogo intercultural propiciado pelas atividades telecolaborativas, na seção a seguir apresentamos algumas conclusões acerca do estudo realizado, bem como alguns possíveis encaminhamentos.

\section{Algumas conclusões e encaminhamentos}

Como dito anteriormente, o objetivo do nosso estudo foi de discutir o potencial da telecolaboração como facilitadora do diálogo intercultural nas aulas de inglês do IFC. Os resultados do estudo revelaram que as atividades telecolaborativas, dada a natureza dialógica da telecolaboração (O'DOWD, 2003, 2019, 2021; THORNE, 2006; BELZ, 2007; HELM, 2016), permitiram a discussão em inglês de diferentes assuntos entre pessoas de diferentes horizontes culturais.

Para Kenski (2011, p. 217), o desenvolvimento de atividades pedagógicas por meio das TICs implica "a possibilidade de transpor os limites físicos e temporais das salas de aula e alcançar as pessoas que querem, têm interesse e estão conectadas no mesmo desejo de aprender". Visto por este prisma, e com base nos resultados do nosso estudo, é possível dizer que a telecolaboração favorece intercâmbios linguísticos - bem como o conhecimento de outros referentes culturais -, para muito além das fronteiras da sala de aula.

É válido esclarecer que a geração dos dados para o nosso estudo se deu em 2019, isto é, anteriormente ao contexto da pandemia de COVID-19 que teve início no ano de 2020. Com efeito, pode-se dizer que tal contexto acarretou, como um todo, um expressivo avanço no uso das TICs para a educação. A este respeito, Hildeblando Júnior e Finardi (2020) enfatizam que o referido avanço "evidenciou mais do que nunca o fato de que vivemos em um mundo cada vez mais interconectado onde o limite entre o global-social/local-individual é poroso e interdependente" (p. 255). Ainda, O'Dowd (2021, p. 6) entende que, durante a pandemia, passou a considerar-se "como as iniciativas de aprendizagem em telecolaboração poderiam ser efetivamente integradas nos currículos" 23 .

Em face da realidade que se instaurou em 2020 e com base nos resultados do nosso estudo, sugerimos que projetos de telecolaboração possam, no porvir, constituir uma valiosa oportunidade para a prática

${ }_{23}$ Citação original: "how telecollaborative learning initiatives could be effectively integrated into curricula". 
do inglês não somente no IFC, mas também em outras instituições educacionais, além de promover o diálogo intercultural. De fato, Schaefer, Heemann e Belli (2017, p. 241) asseveram que "a telecolaboração pode contribuir tanto para o desenvolvimento linguístico quanto para a abordagem intercultural". Ainda, acreditamos que os resultados do nosso estudo convirjam, de alguma forma, com a consideração de O'Dowd (2021) apresentada no parágrafo anterior, no sentido de que as atividades telecolaborativas, no nosso estudo, revelaram-se como uma maneira possível de se implementar a telecolaboração no currículo de língua inglesa do Instituto Federal Catarinense.

Concordamos com Byram (1997), Byram, Gribkova e Starkey (2002), Deardorff (2004), Kramsch (2006) e Wagner e Byram (2017) que a telecolaboração pode contribuir para o desenvolvimento da interculturalidade dos estudantes. Efetivamente, Byram (1997) ressalta a necessidade de tal desenvolvimento nas aulas de língua estrangeira. Visto por esse prisma, acreditamos que atividades telecolaborativas, mediante o diálogo intercultural, tenham o potencial de fomentar nos alunos o entendimento da diversidade cultural, bem como a capacidade para reflexão crítica acerca de temas políticos e sociais. Do mesmo modo, as referidas atividades podem ser favoráveis à discussão de valores culturais, atitudes e visões de mundo, propiciando o debate acerca de questões globais assim como de questões locais, aspectos indispensáveis para um cidadão intercultural (BYRAM et al., 2016).

Além de interações por e-mail, como foi o caso em nosso estudo, existem diferentes possibilidades para que projetos telecolaborativos sejam implementados em aulas de inglês, bem como de outras línguas. Uma delas, de acordo com Schaefer e Heemann (2019), é através da utilização do Skype e de plataformas como The Evaluate Project e Schoology, que permitem o diálogo intercultural entre instituições educacionais no exterior.

Defendemos também que atividades telecolaborativas futuras podem desempenhar um papel importante no processo de internacionalização das instituições de educação. Schaefer, Heemann e Belli (2017, p. 239) apontam que a telecolaboração se configura como "um instrumento de conexão com o mundo, capaz de promover encontros interculturais entre os estudantes participantes de programas de mobilidade acadêmica". Com efeito, a mobilidade acadêmica é uma "estratégia inerente aos processos de internacionalização" (BARANZELI; MOROSINI; WOICOLESCO, 2020, p. 257); porém, ela não atinge grande 
V. 11 (3) 170-187 set-dez 2021

parte da população acadêmica, sendo necessário que alternativas sejam buscadas para que mais pessoas possam ter oportunidades de vivenciar um currículo internacionalizado. Um caminho seria, segundo Heemann, Schaefer e Sequeira (2020), a Internacionalização em Casa, o que implica gerar, pela telecolaboração, oportunidades interculturais em um contexto doméstico, bem como fomentar a internacionalização no âmbito interno da própria instituição educacional.

Por último, mas não menos importante, percebe-se que, para professores de inglês, proporcionar a seus alunos o contato com outros falantes da língua mostra-se, muitas das vezes, como um desafio. Neste sentido, e tendo em vista que as TICs favorecem o referido contato (O'DOWD, 2006, 2021), a telecolaboração está a serviço dos professores, que podem mediar o encontro entre estudantes, línguas e culturas, incorporando sua ação pedagógica com práticas e contatos mais significativos.

\section{Referências}

ALCICI, S. A.R. A Escola na sociedade moderna. In: ALMEIDA, N. A. et al. (Orgs.). Tecnologia na escola: abordagem pedagógica e abordagem técnica. São Paulo: Cengage, 2014.

ALMEIDA, N. A. Os diferentes aspectos da linguagem na comunicação. In: et al. (Orgs.). Tecnologia na escola: abordagem pedagógica e abordagem técnica. São Paulo: Cengage learning, 2014.

BARANZELI, C.; MOROSINI, M.C.; WOICOLESCO, V.G. "A chave está na troca" - estudantes de mobilidade como vetores da internacionalização em casa. Revista Série-Estudos, v.25, n.53, p. 253-274, jan/abr 2020.

BARRETT, M. et al. Developing Intercultural Competence through Education. Strasbourg: Council of Europe, 2013.

BELZ, J. A. The development of intercultural communicative competence in telecollaborative partnerships. In: O'DOWD, R. (Org.). Online Intercultural Exchange: an introduction for foreign language teachers. Clevedon: Multilingual Matters, 2007. p. 127-166.

BOLZAN, D. B. Colaboração na produção escrita em segunda língua: uma proposta de revisão por pares para a aula de língua inglesa em uma escola com currículo bilíngue. 219 f. tese (Doutorado em Linguística Aplicada) Programa de Pós-Graduação em Letras, Universidade Federal do Rio Grande do Sul, Porto Alegre, 2016.

BYRAM, M. Teaching and assessing intercultural communicative competence. Clevedon: Multilíngual Matters, 1997.

BYRAM, M.; GRIBKOVA, B.; STARKEY, H. Developing the intercultural dimension in language teaching: a practical introduction for teachers. 
Strasbourg, FR: Council of Europe, 2002. Disponível em: https://rm.coe. int/16802fc1c3. Acesso em: 24 nov. 2020.

BYRAM, M. The Cultnet Intercultural Citizenship Project. In: O'DOWD, R.; LEWIS, T. (Eds.), Online Intercultural Exchange: policy, pedagogy, practice. New York, Routledge, p. 256-262, 2016.

BYRAM, M. et al. From principles to practice in education for intercultural citizenship. Bristol, UK: Multilingual Matters, 2016.

CANALE, M. De la competencia comunicativa a la pedagogía comunicativa del lenguaje. In: CÀNAVES, M. L. (Coord.). Competencia Comunicativa, documentos básicos en la enseñanza de lenguas extranjeras. Peñalara: Edelsa, 1995. p. 63-82.

DEARDORFF, D. K. Internationalization: in search of intercultural competence. International Educator, v.13, n. 3, p. 13-15, 2004.

EROPEAN COMMISSION. The Digital Education Action Plan (21-27). 2020. Disponível em: https://ec.europa.eu/education/sites/default/files/documentlibrary-docs/deap-communication-sept2020 en.pdf Acesso em: 30 jan. 2021.

FURSTENBERG, G. The Cultura exchange program. In: O'DOWD, R.; LEWIS, T. (Eds.). Online intercultural exchange: policy, pedagogy, practice. New York, Routledge, 2016. p. 248-255.

HEEMANN, C.; SCHAEFER, R.; SEQUEIRA, R. M. (2020). O potencial da telecolaboração para o desenvolvimento da Competência Intercultural no contexto da Internacionalização em Casa. In: LEFFA, V.J. et al. (Eds.). Tecnologias e ensino de línguas: uma década de pesquisa em Linguística Aplicada. Santa Cruz do Sul: EDUNISC, 2020. p.129-152.

HELM, F. Facilitated dialogue in Online Intercultural Exchange. In: O'DOWD, R.; LEWIS, T. (Eds.). Online intercultural exchange: policy, pedagogy, practice. New York: Routledge, 2016. p.150-172.

HILDEBLANDO JÚNIOR, C. A.; FINARDI, K. R. Telecolaboração e internacionalização do ensino superior: reflexões a partir da pandemia covid-19. Revista Intercâmbio, v. 45, p. 254-278, 2020.

KELLY, M. Foreword. In: PHIPPS, A.; GONZALEZ, M. (Eds.) Modern Languages: Learning and Teaching in an Intercultural Field. London: Sage Publications, 2004. p. xi-xiii.

KENSKI, V.M. Formação/ação de professores: a urgência de uma prática docente mediada. In: PIMENTA, S. G.; ALMEIDA, M. I. (Orgs.). Pedagogia universitária: caminhos para a formação de professores. São Paulo: Cortez, 2011. p. 213-228.

KRAMSCH, C. From Communicative Competence to Symbolic Competence. The Modern language Journal, V.90, n. 2, p. 249-252, 2006.

O'DOWD, R. Understanding "the other side": Intercultural learning in a Spanish-English e-mail exchange. Language Learning \& Technology, v. 7, n. 2, p. $118-144,2003$. 
V. 11 (3) 170-187 set-dez 2021
O'DOWD, R. The use of videoconferencing and e-mail as mediators of intercultural student ethnography. In: BELZ, J.A.; THORNE, S. (Eds.). Internetmediated intercultural Foreign Language Education. Boston, MA: Heinle and Heinle, 2006. p. 86-120.

O'DOWD, R. Online Intercultural Exchange: an introduction for foreign language teachers. Clevedon: Multilingual Matters, 2007.

O'DOWD, R. Telecollaboration and CALL. In: THOMAS, M; REINDEERS, H.; WARSCHAUER, M. (Eds.). Contemporary computer-assisted language learning. London: Bloomsbury Academic, 2013.

O'DOWD, R; LEWIS, T. Online Intercultural Exchange: policy, pedagogy, practice. London: Routledge, 2016.

O'DOWD, R. A transnational model of virtual exchange for global citizenship education. Language Teaching, v. 53, n. 4, p. 1-14, 2019.

O'DOWD, R. "Virtual exchange: moving forward into the next decade". Computer Assisted Language Learning, v. 34, n. 3, p. 209-224, 2021.

RAMIRO, M. S. S. Competencia Comunicativa Intercultural. Revista Perspectiva. Consejería de Educación de la Junta de Andalucía, n.13, julio de 2007.

RODRIGUES, D. G. A articulação língua-cultura na coconstrução da competência intercultural em uma parceria de Teletandem (português/ espanhol). $187 \mathrm{f}$. dissertação (Mestrado em Estudos Linguísticos) - Instituto de Biociências, Letras e Ciências Exatas, Universidade Estadual Paulista Júlio de Mesquita Filho, São José do Rio Preto, 2013.

SEHNEM, P. R.; LUNA, J. M. F. Estratégias para o ensino de espanhol como atividade na escola pública. Revista Entretextos, Londrina: UEL, v.13, n. 1, p. 97-115, 2013.

SEHNEM, P. R.; SCHAEFER, R. O ensino de línguas estrangeiras como atividade na educação básica: apresentação de elementos para uma proposta teóricometodológica. In Anais do Congresso Internacional de Educação e Tecnologia e Encontro de Pesquisadores em Educação A Distância (p. 1-6). São Carlos, SP, 2018.

SCHAEFER, R. The co-construction of interculturality in the project teletandem Brazil: foreign languages for all. $597 \mathrm{f}$. Tese. (Doutorado em Estudos Linguísticos e Literários) - Programa de Pós-Graduação em Inglês, Universidade Federal de Santa Catarina, Florianópolis, 2019a. Disponível em: https://repositorio.ufsc. br/handle/123456789/199665. Acesso em: 24 jun. 2020.

SCHAEFER, R. The overcoming of an intercultural misunderstanding in Teletandem. Signum: Estudos da Linguagem, v. 22, n. 3, p. 49-72, $2019 \mathrm{~b}$.

SCHAEFER, R. Facilitating the co-construction of interculturality in teletandem through pedagogical mediation. DELTA: Documentação de Estudos em Linguistica Teórica e Aplicada, v. 36, n. 4, p. 1-29, 2020.

SCHAEFER, R. The Co-Construction of Interculturality Through an Ecological Perspective in Teletandem Activities. Rev. bras. linguist. apl., Belo Horizonte, V. 21, n. 1, p. 1-30, 2021. 
SCHAEFER, R.; LUNA, J. M. F. Negotiation of cultural issues equated to nation in e-mail exchanges. Cadernos de Pós-Graduação em Letras, São Paulo, v. 16, n. 2, p. 52-68, jul./dez. 2016.

SCHAEFER, R.; HEEMANN, C; BELLI, M. O papel da telecolaboração na mobilidade acadêmica. In: LUNA, J.M.F.; SEHNEM, P.R. (Orgs.). O Programa Ciências sem Fronteiras em Avaliação. São Paulo: Pontes Editores, 2017. p. 231-248.

SCHAEFER, R.; HEEMANN, C. Entendimento Intercultural por meio de atividades telecolaborativas. In: FERREIRA, G.R. (Org.) Educação e Tecnologias: experiências, desafios e perspectivas. Ponta Grossa: Atena Editora, 2019. p. 83-87.

SEQUEIRA, R. M. Interculturalidade Crítica e Globalização. In: LUNA, J. M.F. (Org.). Internacionalização do Currículo: educação, interculturalidade, cidadania global. 2.ed. São Paulo: Pontes Editores, 2018. p. 55-70.

TELLES, J. A.; VASSALLO, M. L. Foreign language learning in-tandem: Teletandem as an alternative proposal in CALLT. The ESPecialist, v. 27, n. 2, p. 189-212, 2006.

TELLES, J. A. Learning foreign languages in teletandem: Resources and strategies. DELTA - Revista de Estudos em Linguística Teórica e Aplicada, v. 31, n.3, p. 603-632, 2015.

TELLA, S.; MONONEN-AALTONEN, M. Developing dialogic communication culture in media education: integrating dialogism and technology. Helsinki: Media Education Publications, 1998.

THORNE, S. L. Pedagogical and praxiological lessons from Internet-Mediated Intercultural Foreign Language Education Research. In: BELZ, J. A.; THORNE, S. L. (Orgs.). Internet-Mediated Intercultural Foreign Language Education. Boston: Heinle \& Heinle, 2006. p. 2-30

VELOSO, F. S; ALMEIDA, V. B. A fala facilitadora de dois interagentes no contexto de aprendizagem de LE no Teletandem. In: TELLES, J. A. (Org.). Teletandem: Um contexto virtual, autônomo e colaborativo para a aprendizagem de línguas estrangeiras no século XXI. Campinas: Pontes Editores, 2009. p. 149-168.

WAGNER, M.; BYRAM, M. Intercultural Citizenship. The International Encyclopedia of International Communication, 2017.

WARSCHAUER, M. Telecollaboration in foreign language learning. Second Language Teaching and Curriculum Center. Manoa: University of Hawai'i, 1996. 